\title{
Short-Time Behavior and Criticality of Driven Lattice Gases
}

\author{
Urna Basu, ${ }^{1}$ Valerio Volpati, ${ }^{1}$ Sergio Caracciolo, ${ }^{2}$ and Andrea Gambassi ${ }^{1}$ \\ ${ }^{1}$ SISSA-International School for Advanced Studies and INFN, via Bonomea 265, I-34136 Trieste, Italy \\ ${ }^{2}$ Dipartimento di Fisica, Università degli Studi di Milano and INFN, via Celoria 16, I-20133 Milano, Italy
}

(Received 18 July 2016; revised manuscript received 10 January 2017; published 3 February 2017)

\begin{abstract}
The nonequilibrium short-time critical behaviors of driven and undriven lattice gases are investigated via Monte Carlo simulations in two spatial dimensions starting from a fully disordered initial configuration. In particular, we study the time evolution of suitably defined order parameters, which account for the strong anisotropy introduced by the homogeneous drive. We demonstrate that, at short times, the dynamics of all these models is unexpectedly described by an effective continuum theory in which transverse fluctuations, i.e., fluctuations averaged along the drive, are Gaussian, irrespective of this being actually the case in the stationary state. Strong numerical evidence is provided, in remarkable agreement with that theory, both for the driven and undriven lattice gases, which therefore turn out to display the same short-time dynamics.
\end{abstract}

DOI: 10.1103/PhysRevLett.118.050602

Understanding the emergence of collective behaviors in statistical systems out of equilibrium is one of the major challenges of modern physics. Within a bottom-up approach, simplified lattice models were proposed in the past in order to capture specific aspects of nonequilibrium physics, e.g., the presence of external forces that induce steady currents in a system. The driven lattice gas (DLG) is perhaps the simplest among them: it generalizes the equilibrium lattice gas (LG) [1] to account for the presence of an external driving field $E$ that acts along one lattice direction, biasing the particle jump rates. The DLG was introduced in 1983 [2] and it has since become the paradigm for driven diffusive systems [3,4]. At half filling, for any value of $E$, its stationary state shows a nonequilibrium continuous transition from a disordered to an ordered state at a critical temperature $T_{c}(E)$. In the ordered "phase," particles and holes are separated by an interface parallel to the direction of $E$ [2-4]. The drive also introduces a strong spatial anisotropy, and the observed transition differs from that of the Ising universality class $(E=0)$.

In spite of the apparent simplicity of the model, the critical behavior of the DLG has been a matter of debate for the past three decades. Early field-theoretical studies [5] proposed that the evolution of DLG is effectively described by a mesoscopic Langevin equation with spatial anisotropy and a finite particle current near the critical point. Within this theory (referred to as JSLC, from the authors' names), the critical behavior of the transverse fluctuations turn out to be noninteracting (Gaussian). Earlier numerical studies partially supported this prediction [6-8].

However, some important discrepancies $[9,10]$ cast doubts on this conclusion, and, in fact, an alternative description was introduced [11] according to which, in the DLG with infinitely strong drive (IDLG) $E=\infty$, only anisotropy and not current is the relevant nonequilibrium effect. As a consequence, IDLG is described by the same effective model as the randomly driven lattice gas (RDLG), i.e., a DLG with $E$ randomly changing sign. This proposal was contradicted by subsequent studies $[12,13]$ and by a more careful finite-size scaling analysis $[14,15]$. However, this debate continued with the numerical study in Ref. [16], which found the same finite-size scaling functions in the stationary state for both the IDLG and the RDLG.

The numerical study of the DLG is affected by the particularly severe critical slowing-down, typical of systems with a local conservation law. Moreover, extracting information from numerical data is difficult because of the peculiarities of finite-size scaling in the presence of strong anisotropy [17]. A way to bypass this issue is to study the short-time dynamical relaxation towards the stationary state following a quench to the critical point $[18,19]$. For the DLG, this was done in Ref. [20] (see also Ref. [21]), and the resulting critical exponents turned out to be the same as those of the RDLG, even for finite $E$. However, this conclusion has recently been questioned again in Ref. [22], which revisits the short-time critical dynamics and aging in the IDLG and shows agreement with the JSLC theory.

In this Letter, we show that in these lattice gases, the short-time behavior of "transverse" observables, i.e., of quantities that have been spatially averaged along the direction of $E$, is dictated by an effective Gaussian theory, rather independently of the actual microscopic dynamics. To this end, we perform extensive Monte Carlo simulations of the IDLG, RDLG, and LG, showing that the time evolution of suitably defined transverse order parameters agrees excellently with the prediction of the Gaussian theory with one exception in the LG. We also show that, in the stationary state and in the thermodynamic limit, the IDLG still displays this Gaussian behavior, while the RDLG does not. Accordingly, the critical behavior of 
the driven lattice gases cannot actually be distinguished from studying the short-time dynamics of transverse observables. Our findings actually encompass and reconcile a number of apparently contradictory statements, hopefully settling a 20-years-long debate on a set of paradigmatic models.

Models.-The LG is defined on a $d$-dimensional hypercubic lattice where each site $i$ can be either occupied by one particle or empty, with occupation number $n_{i}=1$ or 0 , respectively. The particles jump randomly to empty nearestneighbor sites with rates $w(\Delta \mathcal{H})=\min \left\{1, e^{-\beta \Delta \mathcal{H}}\right\}$, where $\Delta \mathcal{H}$ is the change in the nearest-neighbor attractive Hamiltonian $\mathcal{H}=-4 \sum_{\{i, j\}} n_{i} n_{j}$ due to the proposed jump and $\beta=1 / T$ is the inverse temperature. At half filling, in the thermodynamic limit and at the critical temperature $T_{c}=$ $2 / \log (1+\sqrt{2})$, the LG undergoes a continuous phase transition belonging to the Ising universality class [23].

The DLG is obtained from the LG by adding a constant field $E$ along one lattice axis, which biases the jump rates as $w(\Delta \mathcal{H}+E l)$, where $l=1,-1,0$ for jumps occurring along, opposite, or transverse to the field. This dynamic leads to a nonequilibrium stationary state when the boundary conditions are periodic along the field direction, as detailed balance is broken. At half filling and in the thermodynamic limit, the DLG shows a phase transition at the critical temperature $T_{c}(E)$ below which particles condense in a single strip with interfaces parallel to the direction of $E$ [3]. For $E \rightarrow \infty$, a particle jump along (opposite to) $E$ is always accepted (rejected). This case is referred to as the IDLG and here we focus on it.

The randomly driven lattice gas $[24,25]$ is a variant of the DLG in which the field $E$ changes sign randomly at each attempted move; for simplicity, we consider below $E= \pm \infty$. Similarly to the DLG, the RDLG at half filling undergoes a continuous transition to a phase-separated configuration.

We perform Monte Carlo simulations on a rectangular periodic lattice of size $L_{\|} \times L_{\perp}$, where $\|$ and $\perp$ denote the directions parallel and orthogonal to the drive, respectively, with "volume" $V=L_{\|} L_{\perp}$; each Monte Carlo step consists of $V$ attempted jumps and it sets the unit of time. The evolution is studied at the critical temperature $T_{c}$ starting from a fully disordered configuration, which is equivalent to a quench from $T=\infty$.

The onset of order in the DLG is typically characterized via the so-called anisotropic order parameter,

$$
m=\langle|\mu|\rangle / V
$$

which is the statistical average $\langle\cdots\rangle$ of the first nonzero transverse mode $\mu=\tilde{\sigma}\left(0,2 \pi / L_{\perp}\right)$, where

$$
\tilde{\sigma}\left(k_{\|}, k_{\perp}\right)=\sum_{x=0}^{L_{\|}-1} \sum_{y=0}^{L_{\perp}-1} e^{i\left(k_{\|} x+k_{\perp} y\right)} \sigma_{x y} .
$$

Here, $\sigma_{x y}=2 n_{x y}-1$ is the spin variable associated with each site $(x, y)$ and $\left(k_{\|}, k_{\perp}\right)=\left(2 \pi n_{\|} / L_{\|}, 2 \pi n_{\perp} / L_{\perp}\right)$ denote the parallel and the transverse wave vectors with integers $0 \leq n_{\|, \perp} \leq L_{\|, \perp}-1$.

An alternative order parameter was introduced in Ref. [20] to measure the average absolute value of the magnetization of the lines parallel to $E$, which can also be expressed as a sum of transverse modes:

$$
O=\frac{1}{V} \sum_{y=0}^{L_{\perp}-1}\left\langle\left|\sum_{x=0}^{L_{\|}-1} \sigma_{x y}\right|\right\rangle=\frac{1}{V}\left\langle\left|\sum_{n_{\perp}=1}^{L_{\perp}-1} \tilde{\sigma}\left(0, \frac{2 \pi n_{\perp}}{L_{\perp}}\right)\right|\right\rangle .
$$

In contrast to the LG, both DLG and RDLG show strong anisotropy in space and the finite-size scaling analysis has to be done at a fixed aspect ratio $S_{\Delta}=L_{\|} / L_{\perp}^{1+\Delta}$. The strength of the anisotropy is characterized by $\Delta=$ $\nu_{\|} / \nu-1$, where $\nu$ and $\nu_{\|}$are the critical exponents of the correlation length along the transverse and parallel directions, respectively. As a result, all the critical exponents (see Table I) depend on the direction [3].

Gaussian theory.-The mesoscopic description of the DLG [5] is based on a Langevin equation for the coarsegrained local particle density $\rho(x, t)$. Near criticality, the evolution of the spin density $\phi(x, t)=2 \rho(x, t)-1$ reads

$$
\begin{aligned}
\partial_{t} \phi= & \alpha\left[\left(\tau-\nabla_{\perp}^{2}\right) \nabla_{\perp}^{2} \phi+\tau_{\|} \nabla_{\|}^{2} \phi+\mathcal{E} \nabla_{\|} \phi^{2}\right] \\
& +u \nabla_{\perp}^{2} \phi^{3}-\nabla_{\perp} \cdot \xi .
\end{aligned}
$$

Here, $\tau$ measures the distance from critical point, $\mathcal{E}$ represents the coarse-grained $E$, and $\xi$ is a white noise with $\left\langle\xi_{i}(x, t) \xi_{j}\left(x^{\prime}, t^{\prime}\right)\right\rangle \propto \delta_{i j} \delta^{d}\left(x-x^{\prime}\right) \delta\left(t-t^{\prime}\right)$, while $\tau_{\|}, \alpha$, and $u$ are inconsequential positive constants. As the only relevant interaction in Eq. (4) is $\mathcal{E} \nabla_{\|} \phi^{2}$, the order parameter $\phi$ at vanishing parallel wave vector $k_{\|}=0$ behaves as in a noninteracting theory [5] and therefore transverse fluctuations are Gaussian [14]. It is then natural to investigate the consequences of this strong prediction on the short-time behavior of transverse modes.

The equation of motion for the amplitude $\tilde{\sigma}_{k} \equiv \tilde{\sigma}(0, k)$ of any transverse mode follows from Eq. (4),

TABLE I. Critical exponents in $d=2$ for the JSLC [5], RDLG [24], and LG [23]. The values listed for the JSLC and the RDLG refer to the transverse exponents; the values for the JSLC and LG are exact, while those for the RDLG are obtained approximately from a series expansion.

\begin{tabular}{lccc}
\hline \hline Exponent & JSLC & RDLG & LG \\
\hline$\Delta$ & 2 & 0.992 & 0 \\
$\beta$ & $1 / 2$ & 0.315 & $1 / 8$ \\
$\nu$ & $1 / 2$ & 0.626 & 1 \\
$\eta$ & 0 & 0.016 & $1 / 4$ \\
$z$ & 4 & 3.984 & $15 / 4$ \\
\hline \hline
\end{tabular}




$$
\frac{d}{d t} \tilde{\sigma}_{k}(t)=-\gamma_{k} \tilde{\sigma}_{k}(t)+i \hat{k} \eta_{k}(t),
$$

where $\hat{k}=2 \sin (k / 2)$ and $\gamma_{k}=\lambda\left(\tau+\hat{k}^{2}\right) \hat{k}^{2} . \lambda$ is a coarsegrained diffusion constant, and $\eta$ is the white noise with $\left\langle\eta_{k}(t) \eta_{k^{\prime}}\left(t^{\prime}\right)\right\rangle=2 \lambda Z V \delta\left(k+k^{\prime}\right) \delta\left(t-t^{\prime}\right)$, where $Z$ is a normalization factor.

We are interested in the case of a quench to the critical point from a disordered configuration; i.e., $\tilde{\sigma}_{k}(t=0)=0$ for all $k$. For this choice, Eq. (5) implies a Gaussian probability distribution for $\tilde{\sigma}_{k}(t)$,

$$
P\left[\tilde{\sigma}_{k}(t), \tilde{\sigma}_{k}^{*}(t)\right] \propto \exp \left(-\frac{\left|\tilde{\sigma}_{k}(t)\right|^{2}}{V \tilde{G}_{\perp}(t, k)}\right),
$$

at any time $t$, where $\tilde{G}_{\perp}(t, k)=\left\langle\left|\tilde{\sigma}_{k}(t)\right|^{2}\right\rangle / V$ is the transverse propagator, which is easily determined from Eq. (5); at the critical point $\tau=0, \tilde{G}_{\perp}(t, k)=Z\left(1-e^{-2 \lambda t \hat{k}^{4}}\right) / \hat{k}^{2}$.

The order parameter $m$ can now be calculated from $P$ by taking the average according to Eqs. (1) and (6):

$$
m(t)=\sqrt{\frac{\pi}{4 V} G_{\perp}\left(t, \frac{2 \pi}{L_{\perp}}\right)} \sim \sqrt{\frac{t}{L_{\|} L_{\perp}^{3}}},
$$

where the last expression indicates the behavior at short times, $t \ll L_{\perp}^{4}$, on large lattices, $L_{\perp} \gg \pi$.

The evolution of $O$ in Eq. (3) can be determined analogously, by noting that the sum of a set of Gaussiandistributed variables is also Gaussian. Accordingly,

$$
O(t)=\sqrt{\frac{\pi}{4} \frac{1}{V} \sum_{n_{\perp}=1}^{L_{\perp}-1} \tilde{G}_{\perp}\left(t, \frac{2 \pi n_{\perp}}{L_{\perp}}\right)} .
$$

For large $L_{\perp}$, the sum over $n_{\perp}$ turns into an integral, which yields $Z L_{\perp}(2 \lambda t)^{1 / 4} / \pi$ and

$$
O(t) \sim t^{1 / 8} / L_{\|}^{1 / 2}
$$

to the leading order for $t \ll L_{\perp}^{4}$. The time dependences predicted in Eqs. (7) and (9) were indeed observed in previous numerical studies of short-time dynamics [20,22].

At criticality, a phenomenological scaling analysis [20,22] for $m$ yields

$$
m\left(t, L_{\|} ; S_{\Delta}\right)=L_{\|}^{-\beta /[\nu(1+\Delta)]} \tilde{f}_{m}\left(t / L_{\|}^{z /(1+\Delta)} ; S_{\Delta}\right) .
$$

Remarkably, the prediction in Eq. (7) of the Gaussian theory is compatible with this scaling, independently of the specific set of values of the critical exponents used, namely, those of JSLC, RDLG, and LG in Table I. This compatibility is a direct consequence of the hyperscaling relation $d+\Delta-2+\eta=2 \beta / \nu$ [3] valid for all three sets, and, therefore, in the presence of Gaussian fluctuations, Eq. (10) at short times is not capable of distinguishing between the various universality classes, contrary to what was assumed in Ref. [22]. The same scaling form for $O$, with a different scaling function $\tilde{f}_{O}$, is compatible with the prediction of Gaussian theory in Eq. (9) only if $\eta=0$, which is exactly (approximately) true for JSLC (RDLG). Accordingly, in contrast with the assumption in Ref. [20], $O$ is also not able to distinguish between these two universality classes. For LG, $\eta \neq 0$, thus Eq. (9) does not hold; however, assuming $O \sim L_{\|}^{-1 / 2}$ [20], we get $O(t) \sim t^{1 / 10}$ from the scaling analysis (see the Supplemental Material [26] for the details).

A useful indicator of possible deviations from Gaussian behavior is the Binder cumulant, which, for conserved systems, can be suitably defined as [15]

$$
g=2-\left\langle|\mu|^{4}\right\rangle /\left\langle|\mu|^{2}\right\rangle^{2},
$$

where $\mu$ is the lowest transverse mode. Indeed, a nonzero value of $g$ signals non-Gaussian transverse fluctuations. The JSLC theory in Eq. (4) predicts that the stationary value of $g$ at the critical point vanishes upon increasing the system size, as it was verified in Ref. [15] for the IDLG.

Below, we test the validity of the theoretical predictions discussed above via Monte Carlo simulations in $d=2$.

IDLG.-The evolution of the IDLG is studied at the critical temperature $T_{c}=3.20$ [2], starting from a fully disordered configuration where both $m$ and $O$ vanish. Figure 1(a) shows $m$ as a function of time $t$ for various geometries with no fixed $\Delta$. The data follow the prediction [Eq. (7)] of the Gaussian theory and an excellent collapse is indeed obtained by rescaling the raw data for $m$ in the inset by $L_{\|}^{1 / 2} L_{\perp}^{3 / 2}$, with a growth $\sim t^{1 / 2}$. Figure 1 (b) shows the evolution of $O$, confirming Eq. (9); the scaling collapse is obtained by plotting $O(t) L_{\|}^{1 / 2}$, which grows as $t^{1 / 8}$.

The evolution of the Binder cumulant $g$ defined in Eq. (11) is shown in Fig. 2(a) for various geometries corresponding to a fixed $S_{\Delta}=2^{-8}$ with $\Delta=2$. Consistently with a Gaussian behavior at short times, $g$ is vanishingly small up to a certain time scaling as $\sim L_{\perp}^{z}$ for large $L_{\perp}$, eventually
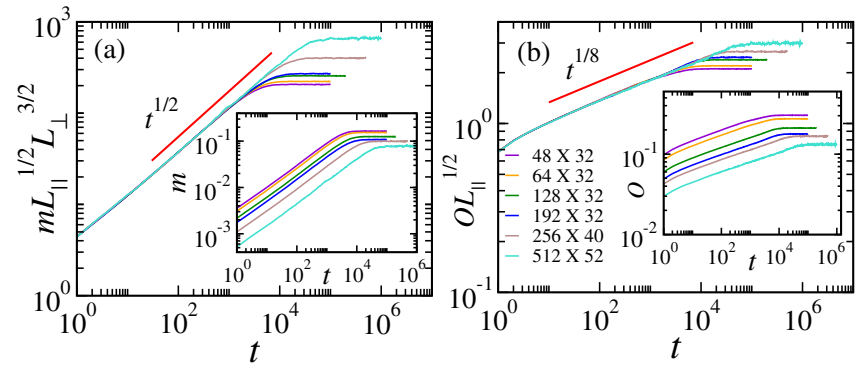

FIG. 1. Short-time behavior of the IDLG. Scaling plot of (a) $m(t)$ and (b) $O(t)$ [see Eqs. (1) and (3)] compared with the predictions in Eqs. (7) and (9), respectively, for different lattice sizes $L_{\|} \times L_{\perp}$. In both panels, the insets show the corresponding unscaled data. 

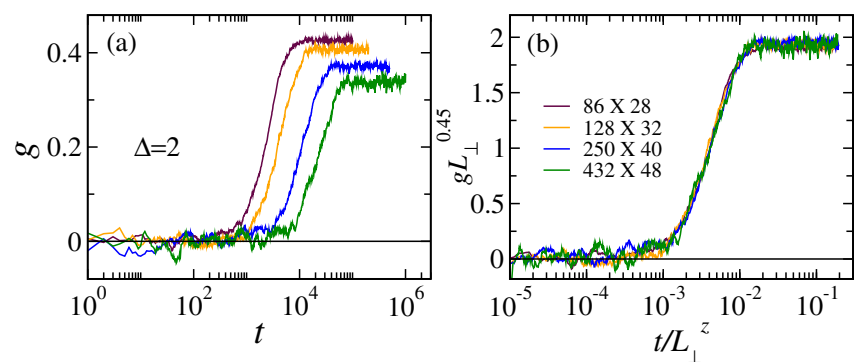

FIG. 2. Binder cumulant $g$ [see Eq. (11)] of the IDLG for the lattice sizes $L_{\|} \times L_{\perp}$ with fixed $S_{\Delta}=2^{-8}$ and $\Delta=2$. The raw data of (a) collapse on a single curve in (b) when $g L_{\perp}^{0.45}$ is plotted vs $t / L_{\perp}^{z}$, with $z=4$.

reaching a stationary value that decreases upon increasing $L_{\perp}$. In fact, it was shown in Ref. [15] that the stationary value of $g \sim L_{\perp}^{-0.45(15)}$. This is verified in Fig. 2(b), which shows the perfect scaling collapse of the same data as Fig. 2(a) multiplied by $L_{\perp}^{0.45}$ and plotted as a function of $t / L_{\perp}^{z}$.

The numerical evidence reported in Figs. 1 and 2 demonstrate that, in the IDLG, the short-time behavior of transverse modes is Gaussian for any finite system, with arbitrary geometry, whereas this is not the case in the stationary state on a finite lattice; however, in the thermodynamic limit, the Gaussian behavior predicted by JSLC is recovered because $g$ vanishes.

RDLG.-The evolution of the RDLG is investigated after a quench to the critical point $T_{c}=3.15$. Figures 3(a) and 3(b) show that the numerical data for $m$ and $O$ follow Eqs. (7) and (9), respectively, as predicted by the Gaussian theory and irrespective of any specific $S_{\Delta}$. In this respect, RDLG and IDLG are indistinguishable.

This Gaussian behavior at short times is also evident from the fact that, independently of the lattice sizes and similarly to the IDLG, $g$ is initially vanishingly small, as shown in Fig. 3(c), and then it increases towards its stationary value. Figure 3(d) shows the same data as in Fig. 3(c) but plotted as a function of $t / L_{\perp}^{z}$ for $\Delta \simeq 1$ and two values of $S_{\Delta}$. In contrast to the IDLG, the stationary value of $g$, for a fixed $S_{\Delta}$, does not change upon increasing the system size, confirming that IDLG and RDLG display different stationary critical behaviors.

LG.-Surprisingly, the IDLG and RDLG show a similar Gaussian behavior at short times, in spite of their different microscopic dynamics. This might be due to the presence of particle conservation in their dynamics. It is then instructive to study critical quenches of the LG, starting from the same initial conditions as in the IDLG and RDLG. LG being isotropic $(\Delta=0)$, the transverse direction is chosen arbitrarily. Figure 4(a) shows $m(t)$ and its scaling according to the prediction $\sim t^{1 / 2}$ of the Gaussian theory for various geometries, showing an excellent collapse. The behavior of $O$, however, is different from Eq. (9), as already pointed out; it shows a $t^{1 / 10}$ growth, while the finite-size
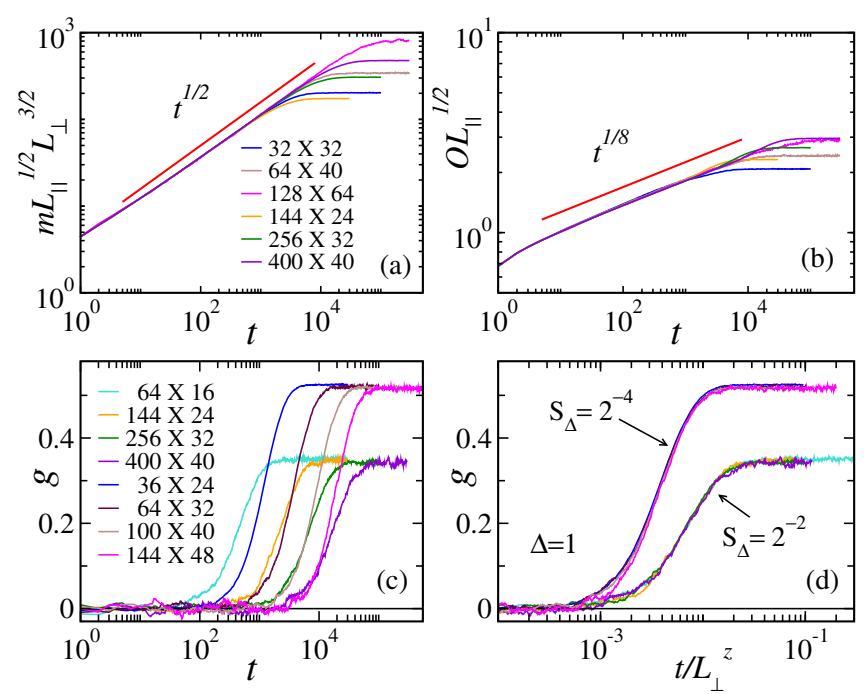

FIG. 3. Evolution of the RDLG. Scaling plot of (a) $m(t)$ and (b) $O(t)$ [see Eqs. (1) and (3)] compared with the predictions in Eqs. (7) and (9), respectively, for different lattice sizes $L_{\|} \times L_{\perp}$. (c) Evolution of the Binder cumulant $g$ in various systems with $\Delta=1$ and two different values of $S_{\Delta}=2^{-4}$ and $2^{-2}$. (d) Same data as in (c) but plotted as a function of $t / L_{\perp}^{z}$, with $z \simeq 4$, according to the RDLG theory.

scaling $\sim L_{\|}^{-1 / 2}$ is still the same as in the other cases. Figure 4(c) shows that, similar to IDLG and RDLG, the Binder cumulant $g$ stays close to zero up to a certain time, which scales $\sim L_{\perp}^{z}$ with $z=3.75$ (see Table I), as can be inferred from the perfect collapse in Fig. 4(d) supporting the Gaussian behavior at short times.
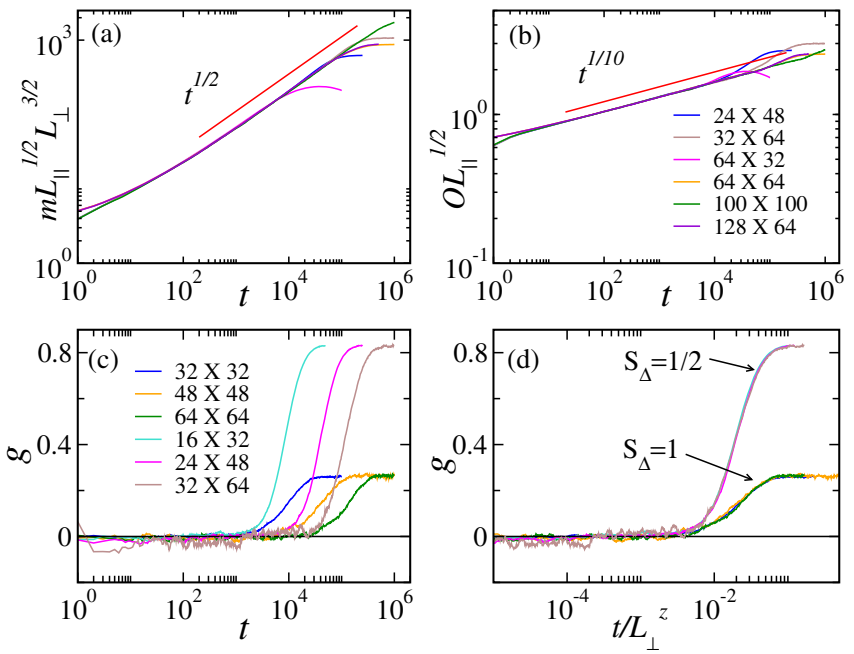

FIG. 4. Evolution of the LG. Scaling plot of (a) $m(t)$ and (b) $O(t)$ [see Eqs. (1) and (3)] compared with the predictions in Eq. (7) and with a growth $\sim t^{1 / 10}$, respectively, for different lattice sizes $L_{\|} \times L_{\perp}$. (c) Evolution of the Binder cumulant $g$ in various systems with $\Delta=0$ and two different values of $S_{\Delta}=1 / 2$ and 1 . (d) Same data as in (c) but plotted as a function of $t / L_{\perp}^{z}$, with $z=3.75$. The resulting scaling curves depend on $S_{\Delta}$. 
Conclusions.-The short-time dynamics of the IDLG, RDLG, and LG after a critical quench has been studied in two dimensions. We provide strong numerical evidence that transverse fluctuations in all these models at short times are governed by a noninteracting effective theory. Presumably, the conservation law renders the dynamics so slow that, before the model-specific correlations of the stationary state are built up, a long lapse emerges in which the behavior is effectively Gaussian [27]. This agrees with the findings of Ref. [22] concerning the evolution of $m \sim t^{0.5}$ and with those of Ref. [20], which showed that $O$ in IDLG and RDLG behave identically at short times. However, our analysis clarifies that this does not imply that these models belong to the same universality class. Our results demonstrate, in fact, that it is not possible to distinguish the critical behavior of IDLG from that of RDLG and, hence, to discriminate between the competing field theories, from the short-time dynamics of transverse observables alone. However, the long-time behavior of the Binder cumulant shows that the two aforementioned models belong to two different universality classes.

U. B. acknowledges the financial support by the ERC under Starting Grant No. 279391 EDEQS.

[1] T. D. Lee and C. N. Yang, Phys. Rev. 87, 410 (1952).

[2] S. Katz, J. L. Lebowitz, and H. Spohn, Phys. Rev. B 28, 1655(R) (1983); J. Stat. Phys. 34, 497 (1984).

[3] B. Schmittmann and R. K. P. Zia, in Statistical Mechanics of Driven Diffusive Systems, Phase Transitions and Critical Phenomena Vol. 17, edited by C. Domb and J. L. Lebowitz (Academic, London, 1995).

[4] R. K. P. Zia, J. Stat. Phys. 138, 20 (2010).

[5] H. K. Janssen and B. Schmittmann, Z. Phys. B 64, 503 (1986); K.-t. Leung and J. L. Cardy, J. Stat. Phys. 44, 567 (1986).

[6] J. L. Vallés and J. Marro, J. Stat. Phys. 43, 441 (1986); 49, 89 (1987).

[7] J.-S. Wang, J. Stat. Phys. 82, 1409 (1996).
[8] K.-t. Leung, Phys. Rev. Lett. 66, 453 (1991); Int. J. Mod. Phys. C 03, 367 (1992).

[9] M. A. Muñoz and P. L. Garrido, Phys. Rev. E 50, 2458 (1994); P. L. Garrido and M. A. Muñoz, Phys. Rev. Lett. 75, 1875 (1995).

[10] J. Marro, A. Achahbar, P. L. Garrido, and J. J. Alonso, Phys. Rev. E 53, 6038 (1996).

[11] P. L. Garrido, F. de los Santos, and M. A. Munoz, Phys. Rev. E 57, 752 (1998); 61, R4683 (2000).

[12] K.-t. Leung, Phys. Rev. E 63, 016102 (2000).

[13] B. Schmittmann, H. K. Janssen, U. C. Täuber, R. K. P. Zia, K.-t. Leung, and J. L. Cardy, Phys. Rev. E 61, 5977 (2000).

[14] S. Caracciolo, A. Gambassi, M. Gubinelli, and A. Pelissetto, J. Phys. A 36, L315 (2003).

[15] S. Caracciolo, A. Gambassi, M. Gubinelli, and A. Pelissetto, J. Stat. Phys. 115, 281 (2004).

[16] A. Achahbar, P. L. Garrido, J. Marro, and M. A. Muñoz, Phys. Rev. Lett. 87, 195702 (2001).

[17] S. Caracciolo, A. Gambassi, M. Gubinelli, and A. Pelissetto, Eur. Phys. J. B 20, 255 (2001); 34, 205 (2003).

[18] H. K. Janssen, B. Schaub, and B. Schmittmann, Z. Phys. B 73, 539 (1989).

[19] B. Zheng, Int. J. Mod. Phys. B 12, 1419 (1998).

[20] E. V. Albano and G. Saracco, Phys. Rev. Lett. 88, 145701 (2002).

[21] S. Caracciolo, A. Gambassi, M. Gubinelli, and A. Pelissetto, Phys. Rev. Lett. 92, 029601 (2004).

[22] G. L. Daquila and U.C. Täuber, Phys. Rev. Lett. 108, 110602 (2012).

[23] G. Mussardo, Statistical Field Theory (Oxford University Press, Oxford, 2009).

[24] B. Schmittmann, Europhys. Lett. 24, 109 (1993).

[25] S. Caracciolo, A. Gambassi, M. Gubinelli, and A. Pelissetto, Phys. Rev. E 72, 056111 (2005).

[26] See Supplemental Material at http://link.aps.org/ supplemental/10.1103/PhysRevLett.118.050602 for the discussion of the behavior of $O$ in the LG.

[27] The idea that the slow conservative dynamics is ultimately responsible for the observed Gaussian behavior at short times is reinforced by the fact that $g$ behaves similarly even when starting from a phase-separated configuration, with the interface lying orthogonal to the drive (data not shown). 